\title{
PERIODIC FUNCTIONS WITH A MULTIPLICATION THEOREM *
}

\section{INTRODUCTION}

Let $f(z)$ represent a uniform analytic function with no essential singularity in the finite part of the plane, and periodic, either simply or doubly. There exists in certain cases a number $m$, different from unity, such that $f(m z)$ is a rational function of $f(z)$. It is desired to determine all of those cases.

Thus a part of our problem is the determination of all elliptic functions which admit of complex multiplication. As far as the writer knows, this question has not been investigated before.

It will be seen that we must have $|m| \geqq 1$, and that the cases with $|m|=1$ are easy to handle. The problem obtains its interest when $|m|$ exceeds unity, and then it can be stated in a form which shows its relation to a result of Poincaré. $\dagger$ He has shown that if $R(z)$ is a rational function such that $R(0)=0$, and that $R^{\prime}(0)$, which we shall denote by $m$, has a modulus greater than unity, there exists a function $f(z)$, meromorphic or entire, such that

$$
f(m z)=R[f(z)] \text {. }
$$

It would be a problem equivalent to the one stated to determine the cases in which $f(z)$ is periodic.

The results are almost negative. We shall state them in detail for $|m|>1$.

If $f(z)$ is simply periodic, $f(z)$ is a linear function, either of $\cos (\alpha z+\beta)$, where $\beta$ is restricted to certain values, or of $e^{\alpha z}$. It will perhaps be considered a noteworthy fact that $f(z)$ cannot be a transcendental function of an exponential, as are, for example, the theta functions.

If $f(z)$ is doubly periodic, and not of the lemniscatic or equianharmonic types, it is of the form $\wp(z+\alpha)$, where $\alpha$ is restricted to certain values. These are the functions regularly used in the theory of complex multiplication.

For the lemniscatic case there are functions of the form $\wp^{2}(z+\alpha)$ in addition to those of the form $\wp(z+\alpha)$, while for the equianharmonic case the forms

* Presented to the Society, February 26, 1921.

† H. Poincaré, Sur une classe nouvelle de transcendantes uniformes, J o u r n a 1 d e M a t h é m a t i q u e s, ser. 3, vol. 55 (1890). 
$\mathscr{P}^{3}(z+\alpha)$ and $\mathscr{P}^{\prime}(z+\alpha)$ are possible. These special cases have been noticed before.*

The possibilities for $|m|=1$ are enumerated rapidly in $\S \mathrm{VII}$.

It will be noticed that we actually use, with respect to $f(m z)$, only the condition that it be a uniform function of $f(z)$.

\section{ProOF That $|m| \geqq 1$}

It is almost obvious that the multiplier $m$ must not be less than unity in modulus. Suppose, in fact, that $|m|<1$. If the value $f(0)$ is assumed $n$ times by $f(z)$ at the point $z=0$, we can determine a neighborhood of the origin in which no value of $f(z)$ is assumed more than $n$ times. Now if $h$ is a period of $f(z)$, and $z_{0}$ any value of $z$, we have

$$
f\left(z_{0}\right)=f\left(z_{0}+h\right)=\cdots=f\left(z_{0}+i h\right)=\cdots,
$$

and, since $f(m z)$ is a uniform iunction of $f(z)$,

$$
f\left(m^{p} z_{0}\right)=f\left[m^{p}\left(z_{0}+h\right)\right]=\cdots=f\left[m^{p}\left(z_{0}+i h\right)\right]=\cdots,
$$

where $p$ is any integer. If we had $|m|<1$, we could, by taking $p$ sufficiently large, bring an unlimited number of points $m^{p}\left(z_{0}+i h\right)$ into the neighborhood taken above, which is impossible, since $f(z)$ is the same for all of those points.

\section{THE AUXILIARY AUTOMORPHISM}

Assuming that $|m|$ exceeds unity, which, in fact, is generally the case in multiplication theorems, we shall deduce a relation which will permit us to show that $f(z)$ cannot take on any value more than twice in a period strip if $f(z)$ is simply periodic, and, except in two cases, not more than twice in a period parallelogram if $f(z)$ is doubly periodic. $†$

Of course if $f(z)$ is doubly periodic, it assumes all values the same number of times in a period parallelogram. If $f(z)$ is simply periodic, and is a rational function of an exponential, it assumes all values the same number of times in a period strip, it being understood that two infinite points are adjoined to the strip. If $f(z)$ is simply periodic and is a transcendental function of an exponential, we know from Picard's theorem that $f(\varepsilon)$ must assume all values, except two, possibly, an infinite number of times in each period strip. These details being mentioned, we shall, in what follows immediately, use the term "fundamental domain" to designate a primitive period strip or a primitive period parallelogram according as $f(z)$ is simply periodic or doubly periodic.

* Weber, Algebra, vol. 3, p. 572.

$\uparrow$ It is understood that we refer to primitzve periods. 
Taking a fundamental domain which contains the origin in its interior, suppose that in it $f(z)$ assumes values more than once.

Let $h$ be any period of $f(z)$. Consider the points

$$
0, \frac{h}{m}, \frac{h}{m^{2}}, \ldots, \frac{h}{m^{p}}, \ldots,
$$

which accumulate at the origin. On the points of this infinite sequence, $f(z)$ must assume an infinite number of distinct values; also, $f^{\prime}(z)$, the derivative of $f(z)$, can vanish for only a finite number of these points. There must consequently exist a point $z_{1}$ of the above sequence, interior to the fundamental domain, at which $f(z)$ is analytic, at which $f^{\prime}(z)$ is not zero, and at which $f(z)$ does not assume an exceptional value, if exceptional values exist; that is, there is another point $z_{2}$ of the fundamental domain for which $f(z)$ has the same value as at $z_{1}$. If $f^{\prime}(0) \neq 0$ and if $f(0)$ is neither infinity nor an exceptional value of $f(z)$, it is simplest to take $z_{1}=0$.

Consider any neighborhood $\Gamma_{1}$ of $z_{1}$ in which $f(z)$ assumes no value more than once; the existence of such a neighborhood follows from the fact that $f^{\prime}\left(z_{1}\right)$ is not zero. There is a neighborhood $\Gamma_{2}$ of $z_{2}$ in which $f(z)$ assumes no value which it does not take on in $\Gamma_{1}$.

Associate with every point of $\Gamma_{2}$ the affix of that point of $\Gamma_{1}$ at which $f(z)$ assumes the same value. This defines a function $\varphi(z)$, analytic in $\Gamma_{2}$. It has a Taylor development

$$
\varphi(z)=z_{1}+\alpha_{1}\left(z-z_{2}\right)+\alpha_{2}\left(z-z_{2}\right)^{2}+\cdots+\alpha_{n}\left(z-z_{2}\right)^{n}+\cdots
$$

We are going to show that $\varphi(z)$ is actually linear.

Let $r$ be an integer so great that of the three points

$$
z_{2}+\frac{h}{m^{r}}, \quad z_{1}+\frac{h}{m^{r}}, \quad \varphi\left(z_{2}+\frac{h}{m^{r}}\right)
$$

the first lies in $\Gamma_{2}$ and the second in $\Gamma_{1}$. Also if $z_{1} \neq 0$, we suppose that $r$ exceeds the integer $p$ used in defining $z_{1}$.

We have

$$
f\left[\varphi\left(z_{2}+\frac{h}{m^{r}}\right)\right]=f\left(z_{2}+\frac{h}{m^{r}}\right) .
$$

Understanding that $f(m z)=R[f(z)]$, let $R_{n}(z)$ denote the $n$th iterate of $R(z)$. We have

$$
f\left(m^{n} z\right)=R_{n}[f(z)]
$$

It is clear then, from (2) and (3), that 


$$
f\left[m^{r} \varphi\left(z_{2}+\frac{h}{m^{r}}\right)\right]=f\left[m^{r}\left(z_{2}+\frac{h}{m^{r}}\right)\right] .
$$

But

$$
\begin{aligned}
f\left[m^{r}\left(z_{2}+\frac{h}{m^{r}}\right)\right] & =f\left(m^{r} z_{2}+h\right)=f\left(m^{r} z_{2}\right) \\
& =R_{r}\left[f\left(z_{2}\right)\right]=R_{r}\left[f\left(z_{1}\right)\right] .
\end{aligned}
$$

We have thus, by (4),

$$
f\left[m^{r} \varphi\left(z_{2}+\frac{h}{m^{r}}\right)\right]=R_{r}\left[f\left(z_{1}\right)\right]=f\left(m^{r} z_{1}\right) .
$$

It is desirable now to show that $m h$ is a period of $f(z)$. This is obvious since

$$
f(z+m h)=R\left[f\left(\frac{z}{m}+h\right)\right]=R\left[f\left(\frac{z}{m}\right)\right]=f(z) .
$$

Similarly $m^{r} h$ is a period, $r$ being any positive integer.

As $r$ exceeds $p$, we see that

$$
f\left(m^{r} z_{1}\right)=f\left(m^{r-p} h\right)=f(0) .
$$

We have by (1)

$$
m^{r} \varphi\left(z_{2}+\frac{h}{m^{r}}\right)=m^{r-p} h+\alpha_{1} h+\alpha_{2} \frac{h^{2}}{m^{r}}+\cdots,
$$

and by (5) and (6), remembering that $m^{r-p} h$ is a period,

$$
f\left(\alpha_{1} h+\alpha_{2} \frac{h^{2}}{m^{r}}+\cdots\right)=f(0)
$$

or any $r$ greater than $p$.

It follows from (7) that $\alpha_{n}=0$ for $n>1$, otherwise, allowing $r$ to pass through all values greater than $p$, we would find an infinite number of distinct points, accumulating at the point $\alpha_{1} h$, for all of which $f(z)$ assumes the value $f(0)$, and $\alpha_{1} h$ would be an essential singularity of $f(z)$. Evidently $\alpha_{1}$ cannot be zero.

Thus a relation

$$
f\left[z_{1}+\alpha_{1}\left(z-z_{2}\right)\right]=f(z)
$$

holds for the region $\Gamma_{2}$. Since the functions which appear in it are analytic, it must hold for the whole plane.

Replacing $z$ by $z / \alpha_{1}+z_{2}$ and $1 / \alpha_{1}$ by $\epsilon$, we may write (8) in the simpler form

$$
f\left(z_{1}+z\right)=f\left(z_{2}+\epsilon z\right) \text {. }
$$

This is the relation referred to at the beginning of the present section. 


\section{FIRST CONSEQUENCES OF THE AUXIIIARY AUTOMORPHISM}

It follows from (9) that if $h$ is any period of $f(z)$ (not necessarily the one used in deriving (9)), $c h$ and $h / \epsilon$ are also periods. Replacing $z$ by $z+h$ in (9), we have

$$
f\left(z_{1}+z+h\right)=f\left(z_{2}+\epsilon z+\epsilon h\right),
$$

and remembering that since $h$ is a period, the first member of (10) equals the second of (9), we see that

$$
f\left(z_{2}+\epsilon z\right)=f\left(z_{2}+\epsilon z+\epsilon h\right),
$$

from which it follows that $\epsilon h$ is a period. Similarly, replacing $z$ by $z+h / \epsilon$ in (9), we find

$$
f\left(z_{1}+z+\frac{h}{\epsilon}\right)=f\left(z_{2}+\epsilon z+h\right)=f\left(z_{1}+z\right),
$$

so that $h / \epsilon$ is a period.

Thus $\epsilon^{r} h, r$ being any positive or negative integer, is a period of $f(z)$. It follows at once that $|\epsilon|=1$, for if not, the moduli of the periods $\epsilon^{r} h$ could be made small at pleasure. Furthermore, $\epsilon$ must be a root of unity, otherwise the affixes of the periods $\epsilon^{r} h$ would be dense along a circle. Finally we cannot have $\epsilon=1$; indeed, the fundamental relation (9) shows that in that case $z_{2}-z_{1}$ would be a period, which is certainly impossible, since $z_{1}$ and $z_{2}$ are both interior to a primitive period strip or to a primitive parallelogram.

These facts in hand, it will not be hard to show that if $f(z)$ is simply periodic $\epsilon$ equals -1 , and that if $f(\varepsilon)$ is doubly periodic, $\epsilon$, if not -1 , is either a third, fourth or sixth root of unity.

The truth of the statement for the simply periodic case follows from the fact that if $h$ is a period of smallest modulus, $c h$, being also a period, must be $-h$.

For the doubly periodic case the proof depends on the proper choice of a primitive pair of periods. We proceed as follows. Of all the periods of $f(z)$, there are two or more which have a smallest modulus. Let one of these be taken as a first period $2 \omega_{1}$. Rejecting all real multiples of $2 \omega_{1}$, there are two or more of those which remain which have a smallest modulus. Of these we choose one, calling it $2 \omega_{3}$. Then $2 \omega_{1}$ and $2 \omega_{3}$ are a primitive pair of periods. For let a parallelogram be constructed with one vertex at the origin and with sides $2 \omega_{1}$ and $2 \omega_{3}$. If these periods were not primitive, the parallelogram would contain the affixes of other periods, of which at least one would have a modulus less than $\left|2 \omega_{1}\right|$ or less than $\left|2 \omega_{3}\right|$.

Consider the case where $\epsilon$ is not -1 . One of the four numbers

$$
\epsilon,-\epsilon, 1 / \epsilon,-1 / \epsilon \text {, }
$$


has a positive argument not greater than $\pi / 2$. Let that number be denoted by $\alpha$ and its argument by $\theta$. Having chosen $2 \omega_{1}$ we can take $2 \alpha \omega_{1}$ as the second period.

If $\theta=\pi / 2$ the period parallelogram is a square and we have the lemniscatic case.

If $\theta<\pi / 2$, the periods $2 \omega_{1}$ and $2 \alpha^{2} \omega_{1}$ will form a primitive pair. Since any two primitive period parallelograms have the same area, the angle $2 \theta$ of the parallelogram formed with $2 \omega_{1}$ and $2 \alpha^{2} \omega_{1}$ must be supplementary to the angle $\theta$ in the parallelogram formed with $2 \omega_{1}$ and $2 \alpha \omega_{1}$. We have thus $\theta=\pi / 3$, which shows that we are in the equianharmonic case.

\section{The NON-EXCEPTIONAL CASES With $|m|>1$}

If $f(z)$ is not an elliptic function of the lemniscatic or equianharmonic types, we must have $\epsilon=-1$. It follows that $f(z)$ cannot take on a value more than twice in a fundamental domain, for if $f\left(z_{1}\right)=f\left(z_{2}\right)=f\left(z_{3}\right), z_{1}$ being taken as a period divided by a power of $m$, and $z_{2}$ and $z_{3}$ being any two other points in the same fundamental domain with $z_{1}$, we must have, by (9),

$$
f\left(z_{1}+z\right)=f\left(z_{2}-z\right)=f\left(z_{3}-z\right)
$$

which leads to the absurdity that $z_{3}-z_{2}$ is a period.

Here are two simple observations which will make easy the enumeration of the functions $f(z)$.

If $f(m z)=R[f(z)]$, we have also, letting $F(z)=f(a z)$ where $a$ is any constant,

$$
F(m z)=R[F(z)] \text {. }
$$

Thus, for the simply periodic case, we may suppose that the period is $2 \pi$; we will secure complete generality by multiplying the argument in $f(z)$ by an arbitrary constant.

Also, if $\varphi(z)$ is any linear function

$$
\varphi(z)=\frac{a z+b}{c z+d}
$$

and $\varphi^{-1}(z)$ its inverse, we have

$$
\varphi^{-1} f(m z)=\varphi^{-1} R \varphi\left[\varphi^{-1} f(z)\right],
$$

so that from any function with a multiplication theorem, we can derive others, perhaps simpler ones, which also have multiplication theorems.

Suppose then that $f(z)$ takes on all values twice in a fundamental domain. According to (9) we have

$$
f\left(z_{1}+z\right)=f\left(z_{2}-z\right)
$$


$z_{1}$ and $z_{2}$ being properly chosen. Replacing $z$ by $z-\left(z_{1}-z_{2}\right) / 2$ and $\left(z_{1}+z_{2}\right) / 2$ by $u$, we have

$$
f(u+z)=f(u-z) .
$$

Consider the case where $f(z)$ is simply periodic with the primitive period $2 \pi$. We see from (11) that $f(u+z)$, being even with respect to $z$, and taking on all values twice in a strip, is a linear function, integral or fractional, of cos $z$. What is the same, $f(z)$ is a linear function of $\cos (z-u)$. Then some linear function of $f(z)$, which also has a multiplication theorem, equals $\cos (z-u)$. It remains to determine when $\cos (m z-u)$ is a rational function of $\cos (z-u)$. Of course $m$ must be an integer, since $2 m \pi$ has to be a period of $\cos (z-u)$. Furthermore, since $\cos \left(z_{1}-u\right)=\cos \left(z_{2}-u\right)$ when

$$
z_{1}-u+z_{2}-u \equiv 0 \quad(\bmod 2 \pi),
$$

we must have, in that case,

$$
m \varepsilon_{1}-u+m \varepsilon_{2}-u \equiv 0 \quad(\bmod 2 \pi) .
$$

Multiplying (12) by $m$ and subtracting the result from (13), we find

$$
u=\frac{k \pi}{m-1}
$$

where $k$ is an integer.

If $f(z)$ assumes no value more than once in a period strip, it must be a linear function of $e^{i s}$.

Summing up, we see that the only simply periodic functions with a multiplication theorem are the functions

$$
\frac{a \cos (\alpha z+\beta)+b}{c \cos (\alpha z+\beta)+d}, \quad \beta=\frac{k \pi}{m-1},
$$

and the functions

$$
\frac{a e^{\alpha \mathbf{z}}+b}{c e^{\alpha z}+d}
$$

The nature of the constants needs no explanation.

Take now the case in which $f(z)$ is doubly periodic, and of the second order.* If the periods of $f(z)$ are $2 \omega_{1}$ and $2 \omega_{3}$, we see by (11) that $f(u+z)$, being even and of the second order, is a linear function of $\&\left(z \mid \omega_{1}, \omega_{3}\right)$. That is, some linear function of $f(z)$ is equal to $\&(z-u)$. We get here nothing more than the ordinary

* We shall see in $\$ \mathrm{VI}$ that if $f(\mathrm{~s})$ is of the second order, the relation (11) holds, even if $f(s)$ is lemniscatic or equianharmonic. 
formulas for the multiplication of the argument, in $\wp(z+\alpha)$, either by a real or by a complex factor. We have

$$
f(z)=\frac{a \wp(z+\alpha)+b}{c \S(z+\alpha)+d},
$$

the multiplier $m$ satisfying the congruences

$$
2 m \omega_{1} \equiv 0,2 m \omega_{3} \equiv 0 \quad\left(\operatorname{modd} 2 \omega_{1}, 2 \omega_{3}\right),
$$

and the constant $\alpha$ being given by the equation

$$
\alpha=\frac{h \omega_{1}+k \omega_{3}}{m-1}
$$

where $h$ and $k$ are any integers.

\section{The SPECIAL CASES WITH $|m|>1$}

Suppose that in the relation (9),

$$
f\left(z_{1}+z\right)=f\left(z_{2}+\epsilon z\right)
$$

$\epsilon$ is a primitive $n$th root of unity. Then the order of $f(z)$ is at least $n .^{*}$ For replacing $z$ by $z+\left(z_{1}-z_{2}\right) /(\epsilon-1)$ and $\left(\epsilon z_{1}-z_{2}\right) /(\epsilon-1)$ by $u$, we find

$$
f(u+z)=f(u+\epsilon z) .
$$

This equation shows that in the development of $f(u+z)$ in powers of $z$ at the origin, the exponents are all multiples of $n$. Consequently the value $f(u)$ is assumed at least $n$ times by $f(z)$ at the point $u$.

If $f(z)$ is of the lemniscatic type, and of order greater than two, it is necessarily of order four. Indeed, if $f(z)$ is of order $n$, there exist relations

$$
f\left(z_{1}+z\right)=f\left(z_{2}+\epsilon_{2} z\right)=\cdots=f\left(z_{n}+\epsilon_{n} z\right),
$$

where $\epsilon_{2}, \ldots, \epsilon_{n}$ are fourth roots of unity. We cannot have $\epsilon_{i}=\epsilon_{j}$, else $z_{i}-z_{j}$ would be a period. Hence $n$ cannot exceed 4 . But since only one $\epsilon$ can equal -1 , one of them must be $\pm i$. Hence $n$ is at least four.

Similarly, if $f(z)$ is of the equianharmonic type, and of order greater than 2 , it must be of order 6 or of order 3 according as there is or is not an $\epsilon$ which is a primitive sixth root of unity.

* C.f. the footnote on p. 22. 
Suppose that $f(z)$ is lemniscatic, and of order 4. There must exist a relation

$$
f\left(z_{1}+z\right)=f\left(z_{2}+i z\right),
$$

or, by (14),

$$
f(u+z)=f(u+i z) .
$$

As was seen above, $f(u+z)$ assumes the value $f(u)$ four times for $z=0$, and the exponents in its expansion in powers of $z$ at the origin are all divisible by 4 . Consequently either $f(u+z)$ or $1 /[f(u+z)-f(u)]$ has a pole of order 4 at the origin, with a principal part of the form $c / z^{4}$. In all, we see that some linear function $F(z)$ of $f(u+z)$ has a pole of order 4 at the origin, with $1 / z^{4}$ for principal part. But the function $\wp^{2} z$ is precisely of this type.* Hence $F(z)-\wp^{2}(z)$, having no poles, is a constant. It follows that $f(z)$ is a linear function of $\ell^{2}(z-u)$.

It remains to determine, for the lemniscatic case, when $\phi^{2}(m z+\alpha)$ is a rational function of $\phi^{2}(z+\alpha)$. We must have, first, $m=p+q i$ with $p$ and $q$ integral, and since $\delta^{2} i u=\varnothing^{2} u$ in this case, the congruence

$$
z_{1}+\alpha \equiv i\left(z_{2}+\alpha\right) \quad\left(\operatorname{modd} 2 \omega_{1}, 2 \omega_{3}\right)
$$

must imply the congruence

$$
m z_{1}+\alpha \equiv i\left(m z_{2}+\alpha\right) \quad\left(\operatorname{modd} 2 \omega_{1}, 2 \omega_{2}\right),
$$

so that

$$
\alpha=\frac{2 h \omega_{1}+2 k \omega_{3}}{(m-1)(1-i)}
$$

where $h$ and $k$ are any integers.

The equianharmonic cases can be handled in the same way. It will suffice to state the results.

If $f(z)$ is of the third order, it must be a linear function of $\mathscr{P}^{\prime}(z+\alpha)$. Here $m=p+q e^{2 \pi i / 3}$ with $p$ and $q$ integral, and

$$
(m-1)\left(1-e^{2 \pi i / 3}\right) \alpha \equiv 0 \quad\left(\operatorname{modd} 2 \omega_{1}, 2 \omega_{3}\right) \text {. }
$$

If $f(z)$ is of the sixth order it must be a linear function of $f^{8}(z+\alpha)$, or, what amounts to the same, of the fourth derivative of $8(z+\alpha)$, where

$$
(m-1)\left(1-e^{\pi i / 3}\right) \alpha \equiv 0 \quad\left(\operatorname{modd} 2 \omega_{1}, 2 \omega_{2}\right)
$$

- We might also have used the second derivative of $\wp_{z}$. 


\section{THE CASE OF $|m|=1$}

In the case of $|m|=1$, since if $h$ is a period of $f(z), m h$ is one also, it follows as in $\$ I V$ that $m$ is either -1 or a third, fourth or sixth root of unity. Let $m$ be a primitive $n$th root of unity and let

$$
f(m z)=R[f(z)] .
$$

Since

$$
f\left(m^{n} z\right)=f(z)=R_{n}[f(z)],
$$

the rational function $R(z)$ must be a periodic linear fraction of which the period is $n$, or a divisor of $n$. Subjecting $f(z)$ to a suitable linear transformation, we may suppose that we are in the case where

$$
f(m z)=\epsilon^{r} f(z),
$$

where $\epsilon$ is a primitive $n$th root of unity.

Suppose first that $f(z)$ is simply periodic; we must have $m=-1$. If $=1, f(z)$ is a rational function of $\cos \alpha z$, with $\alpha$ arbitrary. If $\epsilon^{r}=-1, f(z)$ is the product of $\sin \alpha z$ by a rational function of $\cos \alpha z$.

If $f(z)$ is elliptic, and if $m=-1, f(z)$ is either a rational function of $\varnothing_{z}$, or the product of $\delta^{\prime} z$ by such a rational function.

It is understood that in the cases we are enumerating, any linear function of (z) also has a multiplication theorem.

If $f(z)$ is lemniscatic and if $m$ is not -1 , we may suppose that $m=i$. We must have

$$
f(i z)=i^{r} f(z) \text {. }
$$

If $r=0, f(z)$ is a rational function of $8^{2} z$. If $r=1, f(z)$ is the product of $\mathscr{P}^{\prime} s$ by a rational function of $8^{2} z$. For $r=2$, we multiply the rational function by $\wp_{z}$, and for $r=3$ we multiply it by the third derivative of $\varnothing_{z}$.

The equianharmonic cases are handled the same way. If $m$ is a third root of unity, $f(z)$ is either a rational function of $\varnothing^{\prime} \varepsilon$, or the product of such a rational function by $\wp_{z}$ or one of its derivatives. If $m$ is a primitive sixth root of unity one would use $\varphi^{3} z$ instead of $\mathscr{P}^{\prime} z$.

Colunara UNIVERSITY,

NEW YORK, N. Y. 